\title{
The Negative Symptoms of Schizophrenia: Category or Continuum?
}

\author{
Stefan Kaiser ${ }^{\mathrm{a}, \mathrm{b}} \quad$ Karsten Heekeren $^{\mathrm{a}} \quad$ Joe J. Simon ${ }^{\mathrm{b}}$ \\ ${ }^{a}$ Department of General and Social Psychiatry, Psychiatric University Hospital Zurich, Zurich, Switzerland; \\ bSection of Experimental Psychopathology and Neurophysiology, Department of General Adult Psychiatry, \\ Center for Psychosocial Medicine, Heidelberg, Germany
}

\section{Key Words}

Negative symptoms $\cdot$ Schizophrenia $\cdot$ Affective disorders . Schizotypy $\cdot$ Dimensional diagnostics $\cdot$ Categorical diagnosis

\begin{abstract}
Negative symptoms have been considered to be specific to schizophrenia or a subtype of schizophrenia: the deficit syndrome. In other words, these symptoms have been considered to be categorically different from other forms of human behavior and experience, whether they occur in healthy persons or patients with other psychiatric disorders. In this paper, we advocate a dimensional approach to negative symptoms, which is supported by two main arguments. First, enduring negative symptoms can even be observed in a variety of psychiatric disorders and they are not specific to schizophrenia. Second, we review evidence that negative symptoms show a continuous distribution from apparently healthy subjects to those with a fully developed clinical syndrome. Although the evidence does not allow for a definite decision concerning the dimensional distribution of negative symptoms, it certainly justifies exploring a dimensional approach with respect to its clinical and scientific utility. Understanding negative symptoms as a variation of normal mental processes will strengthen the development of neurocognitive models and treatment approaches.
\end{abstract}

Copyright ๑ 2011 S. Karger AG, Basel
(C) 2011 S. Karger AG, Basel

0254-4962/11/0446-0345\$38.00/0

Fax +4161306 1234

E-Mail karger@karger.ch

www.karger.com
Accessible online at:

www.karger.com/psp

\section{Introduction}

In the original descriptions of schizophrenia, negative symptoms were considered to be defining characteristics of the illness [1]. In the second half of the 20th century, research focused mainly on positive symptoms, but interest in negative symptoms has developed in a very dynamic fashion since the 1980s when a standardized assessment first became available [2]. The term 'negative symptoms' was conceived because these symptoms are thought to reflect the absence or reduction of normal brain function [3]. Regarding negative symptoms of schizophrenia, this reduction manifests itself in phenomena that include avolition, anhedonia, affective flattening and poverty of speech. There is conclusive evidence that negative symptoms contribute significantly to long-term morbidity and poor functional outcome $[4,5]$. At the same time, currently available treatments have at best modest impact on negative symptoms [6]. Thus, negative symptoms are considered to have high priority in treatment development [7].

Kraepelin [1] considered a characteristic disorder of volition to be specific to schizophrenia and did not observe this phenomenon in patients with manic-depressive disorder. In the last 20 years, some authors have emphasized the difference between primary negative symptoms of the schizophrenic illness and secondary symptoms caused by depression, side effects of medication or deprivation $[8,9]$. The former have been conceived

PD Dr. med. Stefan Kaiser

Psychiatric University Hospital Zurich

Lenggstrasse 31

CH-8032 Zurich (Switzerland)

Tel. +41 44384 2630,E-Mail stefan.kaiser@puk.zh.ch 
to be more enduring and particularly difficult to treat. Importantly, these symptoms have been thought to be specific to schizophrenia $[10,11]$. This approach has been taken further, leading to the definition of a deficit syndrome, which largely consists of persistent negative symptoms [12]. It has been suggested that this syndrome reflects a separate disease entity within the schizophrenia spectrum [13]. A concept underlying these distinctions is that primary negative symptoms are specific to schizophrenia or a subtype of schizophrenia and reflect particular neurobiological mechanisms. In other words, the negative symptoms in schizophrenia are considered to be categorically different from other forms of human behavior and experience, whether they occur in 'healthy' persons or patients with other psychiatric disorders.

In this paper, we would like to advocate a different approach to negative symptoms based on dimensional approaches to psychopathology. This approach rests on two main arguments. First, negative symptoms can be observed in a wide variety of neuropsychiatric disorders, in particular on a mood-psychotic disorder continuum, and they are therefore not specific to schizophrenia. Second, negative symptoms show a continuous distribution from variations in apparently healthy subjects over subclinical at-risk states up to full-blown clinical syndromes. A dimensional approach will have important implications for research on the etiology and treatment of negative symptoms. Before we consider these arguments in detail, we briefly review the key components of negative symptoms.

\section{The Structure of Negative Symptoms in Schizophrenia}

Although negative symptoms often appear conjointly, recent research has emphasized their multidimensional structure [14]. This is an important issue because the different dimensions might have different underlying pathophysiological mechanisms and might require different treatment approaches. The most comprehensive assessment instrument for negative symptoms is the Scale for the Assessment of Negative Symptoms (SANS), which consists of five subscales addressing affective flattening, alogia, avolition/apathy, anhedonia/asociality and attentional impairment [2, 7]. Other instruments tend to have a narrower focus and deemphasize some of these subscales. The Positive and Negative Syndrome Scale is also suitable for the assessment of negative symptoms, although problems with the original negative subscale have repeatedly been shown $[15,16]$. These problems can be circumvented when a modified negative subscale or a negative factor score is used [17]. Another prominent instrument is the Schedule for the Deficit Syndrome, which specifically addresses primary and enduring negative symptoms (see discussion of the deficit syndrome concept below) [18]

Recently, the MATRICS (Measurement and Treatment Research to Improve Cognition in Schizophrenia) Consensus Development Conference on Negative Symptoms agreed on a set of relevant dimensions, which include blunted affect, alogia, asociality, anhedonia and avolition [19]. These dimensions form the basis for two assessment instruments, for which initial validation data have recently been published. The resulting Brief Negative Symptom Scale is a 13-item instrument primarily for use in clinical trials [20]. The Clinical Assessment Interview for Negative Symptoms aims at a comprehensive assessment of negative symptoms including objective measures of behavior and subjective patient experience [21].

With respect to relevant scale dimensions, it has to be noted that negative symptoms and cognitive impairment are now conceived of as separate symptom dimensions that are only moderately correlated [22]. The attentional impairment subscale of the SANS has been found to be more closely associated with cognitive impairment and is therefore usually excluded from analyses [23]. For the same reason, newer instruments for the assessment of negative symptoms do not include items specifically addressing cognition.

A number of statistical approaches have been employed to address the structure underlying comprehensive negative symptom assessments $[14,24]$. The individual items can broadly be summarized under two main factors (fig. 1). The factor 'diminished expression' consists of the affective flattening/blunted affect and alogia subscales. The second main factor encompasses the avolition/apathy and anhedonia/asociality subscales and has received a number of designations including amotivation and avolition. However, we suggest that apathy might be the most helpful description. If defined as a quantitative reduction in goal-directed behavior, the term apathy has the advantage not to invoke underlying mechanisms that are difficult to define [25].

It has to be noted that anhedonia in the strict sense does not seem to be a core negative symptom. The experience of pleasure in response to rewarding events has consistently been shown to be preserved in patients with schizophrenia, while they seem less capable of anticipating these experiences [26]. The assessment of anhedonia by interview or questionnaire has generally focused on the latter aspect, which might be the reason why patients 
with schizophrenia consistently have higher scores. For example, the SANS anhedonia items do not primarily address the pleasurable experience itself, but the amount of and interest in certain activities. Therefore, the subsumption of these items rating anticipatory anhedonia under the apathy factor has high face validity. Newer instruments attempt to assess both anticipatory and consummatory anhedonia, but whether these can be reliably differentiated remains to be seen $[20,27]$.

Thus, there is a general consensus on the negative symptom dimensions, which include blunted affect, alogia, asociality and avolition. The role of anhedonia is a matter of current debate. In addition, there is emerging evidence that these dimensions can be subsumed under two factors, namely diminished expression and apathy. It has to be kept in mind that these two broader dimensions are moderately correlated. Whether this type of differentiation will be of clinical value cannot be definitely answered at the moment. However, it already provides a basis for developing neurocognitive accounts of negative symptoms.

A critical issue with respect to the assessment of negative symptoms is how to separate them from depressive and extrapyramidal symptoms. There is clearly an overlap between negative symptoms and depressive symptoms such as retardation and lack of energy [28]. However, it has been convincingly shown that this is not the case when employing the Calgary Depression Scale, which has been specifically developed to assess depression in patients with schizophrenia $[29,30]$. The overlap of negative and extrapyramidal symptoms has repeatedly been shown in earlier studies, but has received less attention with the introduction of modern atypical antipsychotic drugs [31, 32]. However, an assessment of extrapyramidal symptoms should complement studies on negative symptoms even in samples not diagnosed with schizophrenia.

\section{Negative Symptoms along a Mood-Psychotic Disorder Continuum}

The question whether negative symptoms are specific to schizophrenia is a main topic of current discussions about the Kraepelinian dichotomy between dementia praecox and manic-depressive disorders [33]. The validity of a dichotomic classification has been challenged on the grounds of clinical data, but also from a genetic and neuroscientific perspective $[34,35]$. While Kraepelin did not employ the term 'negative symptoms', he clearly considered a disorder of volition as characteristic of schizo-

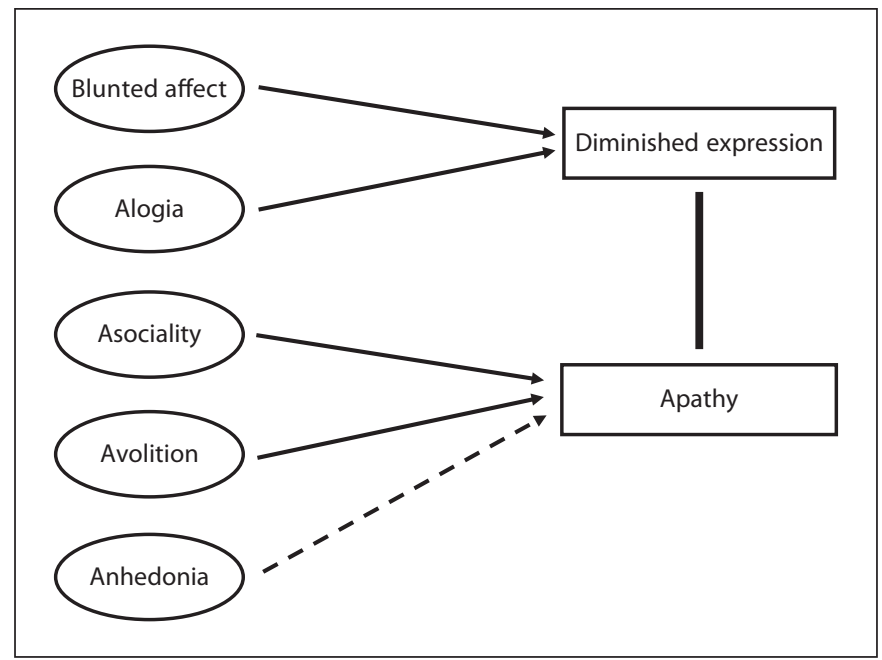

Fig. 1. Overview of the negative symptom subscales that are assessed by current methods. As depicted, these subscales can be subsumed under 2 factors, 'diminished expression' and 'apathy', while the status of anhedonia remains a matter of debate.

phrenia [1]. While the concept of avolition has also been linked to executive dysfunction [36], Kraepelin himself and recent authors have related this concept to reduced motivation for goal-directed behavior rather than its implementation [24]. More recent attempts to differentiate schizophrenia and affective disorders have also relied on the alleged specificity of negative symptoms in addition to first-rank symptoms.

A first group of studies has addressed the question whether negative symptoms occur in affective disorders and whether instruments for assessing these symptoms show a comparable factor structure in these populations. These studies have shown that negative symptoms occur in affective disorders and can be assessed with rating scales originally developed for patients with schizophrenia $[37,38]$. In addition, factor-analytic studies in patients have provided evidence that a negative symptom factor emerges in subjects with affective disorders [39-41]. Most of these studies have employed relatively broad inclusion criteria either according to diagnosis (e.g. schizophrenia + bipolar disorder) or symptom (e.g. at least one psychotic symptom). Despite the variation in inclusion criteria and psychopathological assessment, a negative symptom factor emerges consistently and can be separated from a depressive symptom factor. A comparable factor structure has also been found when only patients with bipolar disorder were investigated [42]. Nevertheless, it is a matter of debate whether negative symptoms can be reliably 
differentiated from depressive symptoms in cross-sectional studies $[38,43]$.

An alternative approach to resolve this discrepancy has suggested that only some negative symptoms might be specific to schizophrenia, while others are not. In a detailed psychopathological analysis, Mundt et al. [44] compared patients with schizophrenia, depression, neurosis and diabetes mellitus. As one would expect, global negative symptoms occurred most frequently in the schizophrenia group, but they were also observed in all the other three groups. Further analysis showed that language disturbance and thought disorder were rather specific to schizophrenia, but core negative symptoms such as social withdrawal, affective flattening and loss of motivation were not.

These problems clearly suggest that other ways of narrowing the definition of negative symptoms might be needed to increase their specificity to schizophrenia. To address this issue, a distinction between primary and secondary negative symptoms was introduced [8]. Primary negative symptoms are considered to be reflections of the schizophrenic disease process, while secondary negative symptoms are caused by depression, side effects of medication or social deprivation. According to this concept, secondary negative symptoms can clearly occur in affective disorders, while primary negative symptoms are considered to be characteristic of schizophrenia [10, 11]. However, a number of studies have shown that it is very difficult to clinically arrive at a reliable distinction between primary and secondary negative symptoms $[45,46]$. Differentiation seems to be feasible in neuroleptic-naïve firstepisode patients, but even in this group the relationship between negative symptoms and other dimensions becomes considerably more complex at discharge [47]. Empirical validation has also come from drug withdrawal paradigms, which are not suitable for clinical practice or even most research settings [9]. These practical problems have led the MATRICS committee on negative symptoms to regard the primary/secondary distinction as not essential for clinical trials targeting negative symptoms [7].

A second distinction has been made between transient and enduring negative symptoms, which are easier to distinguish. Negative symptoms of more than 1-year duration seem to be more specific to schizophrenia regardless of the primary/secondary distinction [43, 48]. However, it has to be noted that even in these studies, up to $40 \%$ of patients with major depressive disorder showed enduring negative symptoms. This has been interpreted as reflecting residual depressive symptoms, but there is mainly indirect evidence for this hypothesis. In a longitudinal study with a 10 -year follow-up, Herbener and Harrow
[49] have shown that although enduring negative symptoms are most common in schizophrenia, they are not specific and often occur in other psychotic and affective disorders. Importantly, the relationship to clinical and outcome variables seems similar in schizophrenia and other disorders [50], which casts some doubt on the residual depression hypothesis. Moreover, the impact of negative symptoms and functional outcome on mood disorders would emphasize their clinical relevance to nonschizophrenic disorders. Brieger et al. [51] employed factor analysis on a broad array of psychopathological and outcome variables in patients with bipolar disorder. They found a functioning/disability factor which concurrently loaded on negative symptoms and low functioning. In conjunction with the respective studies in schizophrenia samples, these studies suggest a high functional relevance of negative symptoms across a mood-psychotic disorder spectrum, but further studies are clearly needed.

In the context of the discussion on the specificity of primary and enduring negative symptoms, the Maryland group suggested that these symptoms might not be specific to schizophrenia in general, but to a subtype of the illness they termed 'deficit syndrome' [12]. Deficit syndrome schizophrenia is conceptualized as being categorically different from nondeficit forms, i.e. it is thought to define a separate disease entity [13]. While there is a considerable body of research employing this concept, we will focus on studies with a primary focus on psychopathology. Few studies have employed taxometric analysis as developed by Meehl to differentiate a categorical from a dimensional distribution of negative symptoms in patients with schizophrenia [52]. Blanchard et al. [53] found support for a distinct patient group with primary and enduring negative symptoms. However, Cuesta et al. [54] have not found stable taxa or classes in a large sample of patients with psychotic disorders. Another critical finding is the occurrence of the deficit syndrome in disorders other than schizophrenia. The original definition of the deficit syndrome requires a diagnosis of schizophrenia. However, several researchers have found a surprisingly high rate of primary and enduring negative symptoms fulfilling all other deficit syndrome criteria in affective disorders [55-57]. Möller et al. [58] found considerably lower rates of the deficit syndrome in patients with affective disorders. Despite the conflicting findings, these results have cast some doubt on the notion that the deficit syndrome only occurs in patients with schizophrenia.

Thus, while the reviewed approaches to negative symptoms have certainly refined the concept, there remains doubt as to whether specificity to schizophrenia can be 
Fig. 2. Multidimensional model of symptom dimensions in the mood-psychotic disorder spectrum according to van Os $[59,60]$. a Example of a patient with high expression on depression and negative symptom dimensions. This constellation could account for depressive patients fulfilling deficit syndrome criteria. b A high expression on the negative (and cognitive) symptom dimensions can occur independently of the psychosis dimension. Thus, a patient with a deficit syndrome would not have more severe symptoms in general, but certainly on the negative symptom dimension.

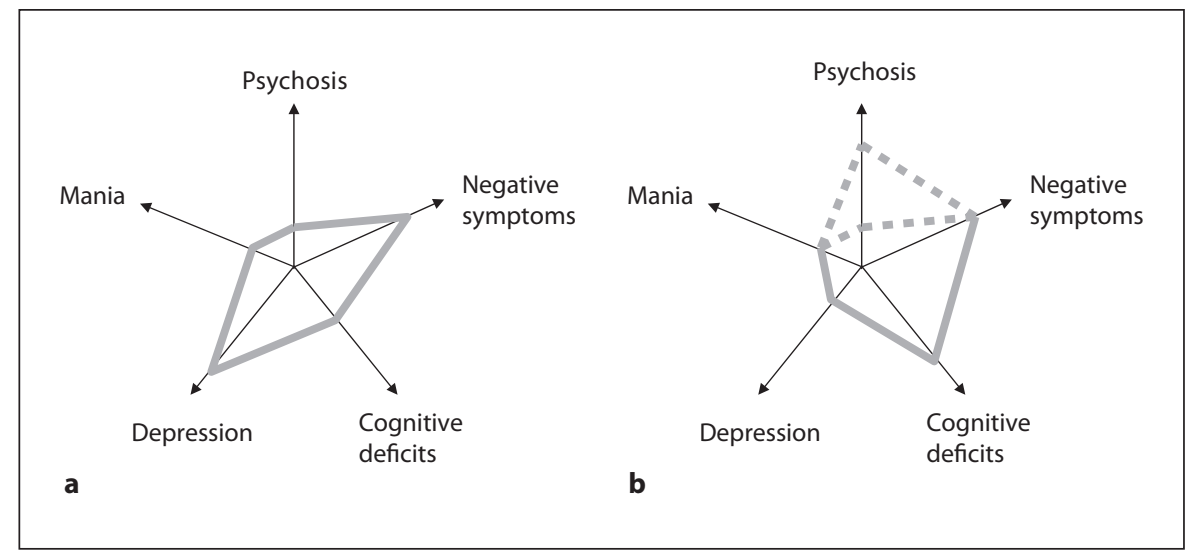

reached. We suggest that these findings - including those relating to the deficit syndrome - can be parsimoniously accounted for in a multidimensional model of the moodpsychotic disorder spectrum. One example of this type of model has been developed by van Os, who defines five (or six) dimensions for this disorder spectrum: positive, negative, cognitive, manic and depressive symptoms $[59,60]$. In this concept, a patient with an affective disorder fulfilling deficit syndrome criteria can be viewed as having a high expression on the affective and negative dimensions, although this co-occurrence might not be common (fig. 2a). Also, the arguments raised by the proponents of the deficit syndrome, claiming that it is not a more severe form of schizophrenia, can be addressed in a multidimensional model. These authors have clearly demonstrated that primary and enduring negative symptoms do not go along with more severe psychotic symptoms and take this as evidence that the deficit syndrome is not just a more severe form of schizophrenia [61]. However, this argument mainly holds against a unidimensional severity spectrum. The dissociation between negative and other symptoms can well be accounted for in a multidimensional approach in which negative symptom expression can be high independently of other symptom dimensions (fig. 2b).

\section{Negative Symptoms in the General Population}

In the previous section, we have discussed to what extent negative symptoms occur in disorders other than schizophrenia, i.e. whether there is a continuum of negative symptoms across disease categories. In the present section, we address the question whether such a continuum can also be described between people diagnosed as having schizophrenia and subjects without any psychiat- ric disorder. Schizophrenic symptoms have been considered the paradigmatic case for experiences and behavior that are fundamentally and categorically different from 'normal' experience and behavior. However, a large body of research has been accumulated over the last 20 years that provides clear evidence for the occurrence of psychotic symptoms in people not diagnosed as having a psychiatric disorder [62].

Johns and van Os [63] describe two conceptual approaches to defining a continuum along a symptom dimension. The first approach holds that in a subdisorder range, symptoms can occur in an attenuated form. These attenuated symptoms are subsumed under the term 'schizotypal'. For example, delusions as a full-blown symptom would be replaced by magical ideation or suspiciousness as attenuated symptoms. In the second approach, there is no qualitative difference between symptoms of patients and symptoms occurring in the general population. The difference is merely quantitative and also regards the associated distress as well as the impact on daily life. This approach has been taken in the Dutch NEMESIS (Netherlands Mental Health Survey and Incidence Study), which demonstrated a high incidence of delusions and hallucinations in the general population [64]. We will now apply both of these approaches to negative symptoms.

Factor-analytic studies of schizotypy questionnaires and interviews have produced a three-factor solution with positive, negative and disorganized components $[65$, 66]. The fact that this factor structure resembles the three-syndrome model of schizophrenia has been interpreted as evidence for continuity between these schizophrenic and schizotypal symptoms. While there is some debate about the disorganization factor, there is compelling evidence for a robust negative schizotypy factor, al- 
though different terms have been applied [67]. In addition, negative schizotypy shows associations with quality of life and neurocognition that are comparable to those observed in patients with schizophrenia $[68,69]$. Another line of research with respect to the continuity debate concerns the relationship between premorbid personality and symptoms of psychotic disorders. Cuesta et al. [70, 71] have shown that negative symptoms are associated with schizoid personality traits prior to illness onset. Their definition of schizoid traits is related to negative schizotypy as used in other studies.

It has to be noted that the symptoms subsumed under negative schizotypy depend on the scale used and the population studied. Critically, in some of these studies, negative schizotypy covers items that are only indirectly related to schizophrenic negative symptoms. Questionnaires will necessarily have a stronger focus on subjective experience than on observed behavior, which is commonly emphasized in rating scales for schizophrenia. But negative factors derived from schizotypy interviews have also included items not directly related to negative symptoms. For example, in the Structured Interview for Schizotypy-Revised, these items encompass social isolation, social anxiety, introversion, restricted affect, referential thinking and suspiciousness [72]. A good example of these challenges is a recent study employing the Oxford-Liverpool Inventory of Feelings and Experiences, a questionnaire that focuses on anhedonia as the sole negative schizotypal symptom [73]. Not surprisingly, the study failed to find a significant correlation between the introversive anhedonia factor and SANS global scores in patients with schizophrenia.

Strong evidence for a continuum comes from the Roscommon Family Study [74]. The authors found that positive and negative symptoms in patients with schizophrenia spectrum disorders predicted corresponding schizotypal symptoms in their relatives. This finding is interesting because it strongly suggests that there is an etiologic continuum. In other words, negative (and positive) symptoms are determined by similar familial etiologic factors in patient and nonpatient groups. In summary, there is strong evidence for a robust negative schizotypy construct. Whether these schizotypal symptoms lie on a continuum with schizophrenic negative symptoms is still a matter of debate and will require studies assessing both symptoms in the same population.

As introduced above, another approach to negative symptoms in the general population would be based on similar symptoms occurring in both patient and nonpatient groups. The group difference would then be regard- ed as a mere question of quantity and associated distress. So far, this line of research has had a strong focus on positive symptoms. Nevertheless, investigations with the CAPE (Community Assessment of Psychic Experiences) have provided evidence for a negative symptom factor in the general population [75]. In addition, the CAPE negative dimension correlates with the Brief Psychiatric Rating Scale negative dimension, providing a first hint for continuity [76]. However, the relationship with more comprehensive negative symptom rating scales such as the SANS has not been addressed.

One major problem in these comparisons is that clinical negative symptom rating scales are interview based and rely at least in part on behavioral ratings by the interviewer. Few studies have employed clinical assessments of negative symptoms in participants drawn from the general population and have generally yielded a relatively low prevalence [77]. However, it is a critical issue whether these clinical rating scales are sensitive to detecting negative symptoms in nonpatient groups. Even though this approach conceives of the symptoms as qualitatively similar, clinical scales might require a high threshold to detect a symptom at all. Therefore, instruments for assessing negative symptoms over the whole range from healthy subjects to patients are urgently needed. This is necessary to expand the preliminary evidence for negative symptoms in the general population that might be qualitatively similar to those in schizophrenia.

\section{Implications of a Dimensional Approach}

In the previous sections, we have presented evidence that negative symptoms occur in disorders other than schizophrenia and that they occur in the general population at least in an attenuated form. Thus, we suggest a dimensional distribution across the mood-psychotic disorder spectrum and along a continuum between manifest disorders, risk status and health (fig. 3). It is clear that the evidence presented cannot constitute definite proof of a dimensional distribution of negative symptoms.

Further diagnostic studies including patient samples across disease categories and in the general population are needed. One important issue for this type of research regards the assessment instruments. The available assessment instruments with a specific focus on negative symptoms were validated for patients with schizophrenia, and this continues to be the case for the instruments resulting from the MATRICS initiative [21]. This might not result in a serious problem with respect to the constructs as- 
sessed, which seem to be stable across disease categories. However, it needs to be addressed whether the available instruments provide adequate sensitivity to mild variations in these symptoms.

Even when studies in appropriate samples are conducted with appropriate scales, there remain methodological problems of arriving at a statistically sound differentiation between dimensional and categorical concepts (see Haslam [78] and Kraemer et al. [79] for discussion). The controversy has been pointedly put as just a different way of looking at the same data, and these authors have suggested that the appropriate approach should be selected according to the respective purpose $[79,80]$. We argue that the evidence reviewed certainly justifies an exploration of the dimensional approach with respect to both its clinical and scientific utility.

First of all, a negative symptom dimension within a multidimensional model of the mood-psychotic disorder spectrum has important implications for research and treatment. Current research has already begun to shift from neurocognitive models of disease entities to models of symptom formation. However, if one takes the multidimensional model seriously, neurocognitive correlates of symptom dimensions should not be investigated within a disease category but across disease categories. A similar argument regards current treatment developments, which turn towards a more differentiated targeting of symptoms instead of a disease like schizophrenia [81]. Here, a shift towards drug development for negative symptoms across the mood-psychotic disorder spectrum could be a promising step.

Second, a dimensional distribution between severe symptom expression and health takes these considerations even further. Importantly, this should lead to a neurocognitive framework for understanding symptoms as variations of normal mental processes [82]. Current research already adopts this approach to some extent by beginning to use common models to address the relationship between symptom or personality dimensions and neurocognitive correlates. However, the empirical work usually addresses these relationships separately in patients or healthy participants. Again, if one takes the dimensional approach seriously, one should address this issue across the spectrum from healthy subjects to those with severe symptom expression. As mentioned above, a critical point for this approach will be the need for assessment instruments that can cover the whole severity spectrum of symptom expression.

Finally, this dimensional approach should also encourage psychotherapeutic approaches to the treatment of

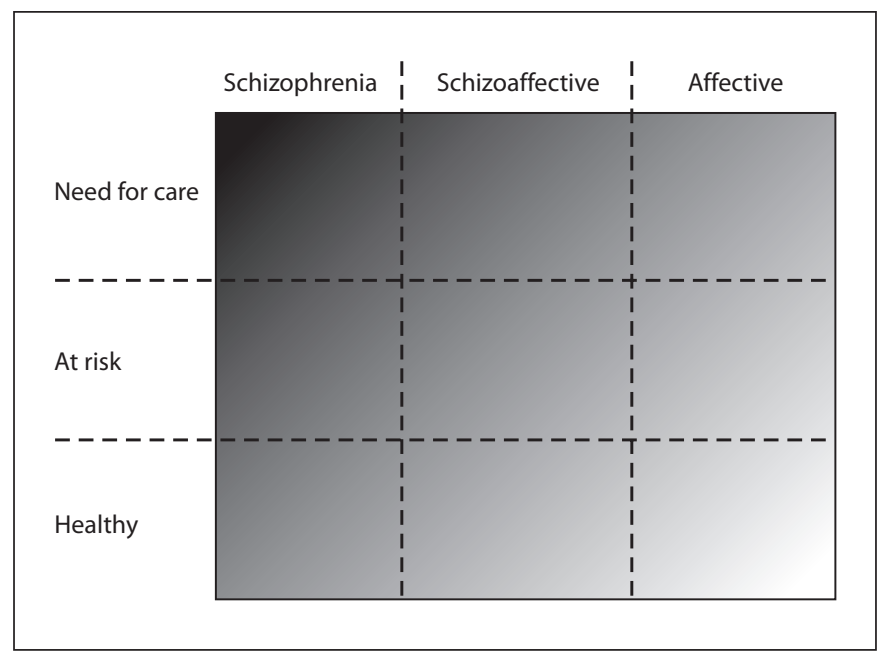

Fig. 3. We propose that negative symptoms are dimensionally distributed across the mood-psychotic disorder spectrum and along a continuum between manifest disorders, risk status and health. Probability and intensity of negative symptoms are marked as darkness of shading. Patients with schizophrenia showing prominent and enduring negative symptoms would be located in the top left of the graph, but nevertheless on a continuum with all other groups. The dashed lines are arbitrary and do not reflect a categorical transition.

negative symptoms. Understanding symptoms as variations of normal processes opens an avenue for cognitive and behavioral interventions. Such an approach has been an important condition for developing cognitive-behavioral therapy for persistent positive symptoms, which are thought to be caused among other things by variations in attributional style, attention allocation and mental state attribution $[83,84]$. The effect of broader cognitive-behavioral therapy programs on negative symptoms has been promising $[85,86]$. However, results of the first largescale trial with negative symptoms as the primary focus do not seem to show specific effects $[87,88]$. This might in part be accounted for by the unexpected improvement in the active control group, and final results have not yet been published. Here, it is a critical issue that - in comparison with cognitive models of delusion formation current neurocognitive models of negative symptoms are rather crude and far less comprehensive. Thus, we are still quite far away from understanding negative symptoms as variations of normal mental processes. Within a dimensional framework, further development of these models will not only be of high scientific interest, but should have a strong impact on approaches to treatment.

Psychopathology 2011;44:345-353 


\section{References}

1 Kraepelin E: Dementia Praecox and Paraphrenia. New York, Krieger, 1919.

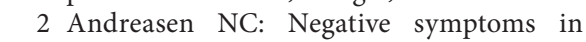
schizophrenia: definition and reliability. Arch Gen Psychiatry 1982;39:784-788.

3 Hughlings-Jackson J: Selected Writings. London, Hodder and Stoughton, 1931.

4 McGlashan TH, Fenton WS: The positivenegative distinction in schizophrenia: review of natural history validators. Arch Gen Psychiatry 1992;49:63-72.

5 Ho BC, Nopoulos P, Flaum M, Arndt S, Andreasen NC: Two-year outcome in first-episode schizophrenia: predictive value of symptoms for quality of life. Am J Psychiatry 1998;155:1196-1201.

-6 Stahl SM, Buckley PF: Negative symptoms of schizophrenia: a problem that will not go away. Acta Psychiatr Scand 2007;115:4-11.

$\checkmark 7$ Kirkpatrick B, Fenton WS, Carpenter WT Jr, Marder SR: The NIMH-MATRICS consensus statement on negative symptoms. Schizophr Bull 2006;32:214-219.

-8 Carpenter WT Jr, Heinrichs DW, Alphs LD: Treatment of negative symptoms. Schizophr Bull 1985;11:440-452.

-9 Kelley ME, van Kammen DP, Allen DN: Empirical validation of primary negative symptoms: independence from effects of medication and psychosis. Am J Psychiatry 1999; 156:406-411.

10 Carpenter WT Jr: Psychopathology and common sense. Biol Psychiatry 1991;29:735737.

11 Tandon R, Greden JF: Negative symptoms of schizophrenia: the need for conceptual clarity. Biol Psychiatry 1991;30:321-325.

-12 Carpenter WT Jr, Heinrichs DW, Wagman AM: Deficit and nondeficit forms of schizophrenia: the concept. Am J Psychiatry 1988; 145:578-583.

13 Kirkpatrick B, Buchanan RW, Ross DE, Carpenter WT Jr: A separate disease within the syndrome of schizophrenia. Arch Gen Psychiatry 2001;58:165-171.

14 Blanchard JJ, Cohen AS: The structure of negative symptoms within schizophrenia: implications for assessment. Schizophr Bull 2006;32:238-245

-15 van der Gaag M, Cuijpers A, Hoffman T, Remijsen M, Hijman R, de Haan L, van Meijel B, van Harten PN, Valmaggia L, de Hert M, Wiersma D: The five-factor model of the Positive and Negative Syndrome Scale. 1. Confirmatory factor analysis fails to confirm 25 published five-factor solutions. Schizophr Res 2006;85:273-279.

16 White L, Harvey PD, Opler L, Lindenmayer JP: Empirical assessment of the factorial structure of clinical symptoms in schizophrenia: a multisite, multimodel evaluation of the factorial structure of the Positive and Negative Syndrome Scale. The PANSS Study Group. Psychopathology 1997;30:263-274.
17 van der Gaag M, Hoffman T, Remijsen M, Hijman R, de Haan L, van Meijel B, van Harten PN, Valmaggia L, de Hert M, Cuijpers A, Wiersma D: The five-factor model of the Positive and Negative Syndrome Scale. 2. A ten-fold cross-validation of a revised model. Schizophr Res 2006;85:280-287.

18 Kirkpatrick B, Buchanan RW, McKenney PD, Alphs LD, Carpenter WT Jr: The Schedule for the Deficit syndrome: an instrument for research in schizophrenia. Psychiatry Res 1989;30:119-123.

19 Blanchard JJ, Kring AM, Horan WP, Gur R: Toward the next generation of negative symptom assessments: the collaboration to advance negative symptom assessment in schizophrenia. Schizophr Bull 2011;37:291299.

20 Kirkpatrick B, Strauss GP, Nguyen L, Fischer BA, Daniel DG, Cienfuegos A, Marder SR: The Brief Negative Symptom Scale: psychometric properties. Schizophr Bull 2011;37: 300-305.

21 Forbes C, Blanchard JJ, Bennett M, Horan WP, Kring A, Gur R: Initial development and preliminary validation of a new negative symptom measure: the Clinical Assessment Interview for Negative Symptoms (CAINS). Schizophr Res 2010;124:36-42.

22 de Gracia Dominguez M, Viechtbauer W, Simons CJ, van Os J, Krabbendam L: Are psychotic psychopathology and neurocognition orthogonal? A systematic review of their associations. Psychol Bull 2009;135:157-171.

23 Vadhan NP, Serper MR, Harvey PD, Chou JC, Cancro R: Convergent validity and neuropsychological correlates of the schedule for the assessment of negative symptoms (SANS) attention subscale. J Nerv Ment Dis 2001;189: 637-641.

24 Foussias G, Remington G: Negative symptoms in schizophrenia: avolition and Occam's razor. Schizophr Bull 2010;36:359369.

25 Levy R, Dubois B: Apathy and the functional anatomy of the prefrontal cortex-basal ganglia circuits. Cereb Cortex 2006;16:916-928.

26 Horan WP, Kring AM, Blanchard JJ: Anhedonia in schizophrenia: a review of assessment strategies. Schizophr Bull 2006;32: 259-273.

27 Gard DE, Kring AM, Gard MG, Horan WP, Green MF: Anhedonia in schizophrenia: distinctions between anticipatory and consummatory pleasure. Schizophr Res 2007;93 253-260.

28 Kulhara P, Avasthi A, Chadda R, Chandiramani K, Mattoo SK, Kota SK, Joseph S: Negative and depressive symptoms in schizophrenia. Br J Psychiatry 1989;154:207-211.

$\checkmark 29$ Addington D, Addington J, Schissel B: A depression rating scale for schizophrenics. Schizophr Res 1990;3:247-251.
30 Rabany L, Weiser M, Weberloff N, Levkovitz Y: Assessment of negative symptoms and depression in schizophrenia: revision of the SANS and how it relates to the PANSS and CDSS. Schizophr Res 2011;126:226-230.

- 31 Lewander T: Neuroleptics and the neuroleptic-induced deficit syndrome. Acta Psychiatr Scand Suppl 1994;380:8-13

32 Dollfus S, Ribeyre JM, Petit M: Objective and subjective extrapyramidal side effects in schizophrenia: their relationships with negative and depressive symptoms. Psychopathology 2000;33:125-130.

33 Craddock N, Owen MJ: The beginning of the end for the Kraepelinian dichotomy. Br J Psychiatry 2005; 186:364-366.

-34 Rosenman S, Korten A, Medway J, Evans M: Dimensional vs categorical diagnosis in psychosis. Acta Psychiatr Scand 2003;107:378384.

35 Purcell SM, Wray NR, Stone JL, Visscher PM, O'Donovan MC, Sullivan PF, Sklar P: Common polygenic variation contributes to risk of schizophrenia and bipolar disorder. Nature 2009;460:748-752.

36 Zec RF: Neuropsychology of schizophrenia according to Kraepelin: disorders of volition and executive functioning. Eur Arch Psychiatry Clin Neurosci 1995;245:216-223.

37 Kulhara P, Chadda R: A study of negative symptoms in schizophrenia and depression. Compr Psychiatry 1987;28:229-235.

38 Galynker II, Cohen LJ, Cai J: Negative symptoms in patients with major depressive disorder: a preliminary report. Neuropsychiatry Neuropsychol Behav Neurol 2000;13:171176.

39 Kitamura T, Suga R: Depressive and negative symptoms in major psychiatric disorders. Compr Psychiatry 1991;32:88-94.

40 Cuesta MJ, Peralta V: Integrating psychopathological dimensions in functional psychoses: a hierarchical approach. Schizophr Res 2001;52:215-229.

41 Serretti A, Olgiati P: Dimensions of major psychoses: a confirmatory factor analysis of six competing models. Psychiatry Res 2004; 127:101-109.

-42 Lindenmayer JP, Bossie CA, Kujawa M, Zhu Y, Canuso CM: Dimensions of psychosis in patients with bipolar mania as measured by the positive and negative syndrome scale. Psychopathology 2008;41:264-270.

43 Bottlender R, Sato T, Groll C, Jäger M, Kunze I, Möller HJ: Negative symptoms in depressed and schizophrenic patients: how do they differ? J Clin Psychiatry 2003;64:954958.

44 Mundt C, Kasper S, Huerkamp M: The diagnostic specificity of negative symptoms and their psychopathological context. Br J Psychiatry Suppl 1989;7:32-36, discussion 3740. 
45 Flaum M, Andreasen N: The reliability of distinguishing primary versus secondary negative symptoms. Compr Psychiatry 1995; 36:421-427.

- 46 Peralta V, Cuesta MJ: Negative parkinsonian, depressive and catatonic symptoms in schizophrenia: a conflict of paradigms revisited. Schizophr Res 1999;40:245-253.

-47 Peralta V, Cuesta MJ, Martinez-Larrea A, Serrano JF: Differentiating primary from secondary negative symptoms in schizophrenia: a study of neuroleptic-naive patients before and after treatment. Am J Psychiatry 2000;157:1461-1466.

-48 Husted JA, Beiser M, Iacono WG: Negative symptoms in the course of first-episode affective psychosis. Psychiatry Res 1995;56: 145-154.

-49 Herbener ES, Harrow M: Longitudinal assessment of negative symptoms in schizophrenia/schizoaffective patients, other psychotic patients, and depressed patients. Schizophr Bull 2001;27:527-537.

50 Herbener ES, Harrow M: Are negative symptoms associated with functioning deficits in both schizophrenia and nonschizophrenia patients? A 10-year longitudinal analysis. Schizophr Bull 2004;30:813-825.

-51 Brieger P, Rottig S, Rottig D, Marneros A, Priebe S: Dimensions underlying outcome criteria in bipolar I disorder. J Affect Disord 2007;99:1-7.

52 Waller NG, Meehl PE: Multivariate Taxometric Procedures. Distinguishing Types from Continua. Thousand Oaks, Sage, 1998.

- 53 Blanchard JJ, Horan WP, Collins LM: Examining the latent structure of negative symptoms: is there a distinct subtype of negative symptom schizophrenia? Schizophr Res 2005;77:151-165.

54 Cuesta MJ, Ugarte MD, Goicoa T, Eraso S, Peralta V: A taxometric analysis of schizophrenia symptoms. Psychiatry Res 2007;150: 245-253.

55 Gerbaldo H, Philipp M: The deficit syndrome in schizophrenic and nonschizophrenic patients: preliminary studies. Psychopathology 1995;28:55-63.

56 Gerbaldo H, Georgi K, Pieschl D: The deficit syndrome in first-admission patients with psychotic and non-psychotic disorders. Eur Psychiatry 1997;12:53-57.

-57 Peralta V, Cuesta MJ: The deficit syndrome of the psychotic illness: a clinical and nosological study. Eur Arch Psychiatry Clin Neurosci 2004;254:165-171.

58 Möller HJ, Bottlender R, Gross A, Hoff P, Wittmann J, Wegner U, Strauss A: The Kraepelinian dichotomy: preliminary results of a 15 -year follow-up study on functional psychoses - focus on negative symptoms. Schizophr Res 2002;56:87-94.

-59 van Os J: 'Salience syndrome' replaces 'schizophrenia' in DSM-V and ICD-11: psychiatry's evidence-based entry into the 21st century? Acta Psychiatr Scand 2009;120: 363-372.
60 van Os J, Kapur S: Schizophrenia. Lancet 2009;374:635-645.

61 Cohen AS, Brown LA, Minor KS: The psychiatric symptomatology of deficit schizophrenia: a meta-analysis. Schizophr Res 2010;118:122-127.

62 van Os J, Linscott RJ, Myin-Germeys I, Delespaul P, Krabbendam L: A systematic review and meta-analysis of the psychosis continuum: evidence for a psychosis pronenesspersistence-impairment model of psychotic disorder. Psychol Med 2009;39:179-195.

63 Johns LC, van Os J: The continuity of psychotic experiences in the general population. Clin Psychol Rev 2001;21:1125-1141.

64 Hanssen M, Bak M, Bijl R, Vollebergh W, van Os J: The incidence and outcome of subclinical psychotic experiences in the general population. Br J Clin Psychol 2005;44:181191.

65 Raine A, Reynolds C, Lencz T, Scerbo A, Triphon N, Kim D: Cognitive-perceptual, interpersonal, and disorganized features of schizotypal personality. Schizophr Bull 1994;20:191-201.

66 Gruzelier JH: The factorial structure of schizotypy. 1. Affinities with syndromes of schizophrenia. Schizophr Bull 1996;22:611620.

67 Vollema MG, van den Bosch RJ: The multidimensionality of schizotypy. Schizophr Bull 1995;21:19-31.

68 Cohen AS, Davis TE 3rd: Quality of life across the schizotypy spectrum: findings from a large nonclinical adult sample. Compr Psychiatry 2009;50:408-414

69 Szöke A, Méary A, Ferchiou A, Trandafir A, Leboyer M, Schürhoff F: Correlations between cognitive performances and psychotic or schizotypal dimensions. Eur Psychiatry 2009;24:244-250.

70 Cuesta MJ, Gil P, Artamendi M, Serrano JF, Peralta V: Premorbid personality and psychopathological dimensions in first-episode psychosis. Schizophr Res 2002;58:273-280.

71 Cuesta MJ, Peralta V, Caro F: Premorbid personality in psychoses. Schizophr Bull 1999; 25:801-811.

72 Vollema MG, Ormel J: The reliability of the Structured Interview for Schizotypy-Revised. Schizophr Bull 2000;26:619-629.

73 Cochrane M, Petch I, Pickering AD: Do measures of schizotypal personality provide non-clinical analogues of schizophrenic symptomatology? Psychiatry Res 2010;176: 150-154.

74 Fanous A, Gardner C, Walsh D, Kendler KS: Relationship between positive and negative symptoms of schizophrenia and schizotypal symptoms in nonpsychotic relatives. Arch Gen Psychiatry 2001;58:669-673.

-75 Stefanis NC, Hanssen M, Smirnis NK, Avramopoulos DA, Evdokimidis IK, Stefanis CN, Verdoux H, van Os J: Evidence that three dimensions of psychosis have a distribution in the general population. Psychol Med 2002;32:347-358.
76 Konings M, Bak M, Hanssen M, van Os J, Krabbendam L: Validity and reliability of the CAPE: a self-report instrument for the measurement of psychotic experiences in the general population. Acta Psychiatr Scand 2006;114:55-61.

77 Emmerson LC, Ben-Zeev D, Granholm E, Tiffany M, Golshan S, Jeste DV: Prevalence and longitudinal stability of negative symptoms in healthy participants. Int J Geriatr Psychiatry 2009;24:1438-1444.

78 Haslam N: Categorical versus dimensional models of mental disorder: the taxometric evidence. Aust NZ J Psychiatry 2003;37:696704 .

79 Kraemer HC, Noda A, O’Hara R: Categorical versus dimensional approaches to diagnosis: methodological challenges. J Psychiatr Res 2004;38:17-25.

-80 Goldberg D: Plato versus Aristotle: categorical and dimensional models for common mental disorders. Compr Psychiatry 2000; 41:8-13

81 Webber MA, Marder SR: Better pharmacotherapy for schizophrenia: what does the future hold? Curr Psychiatry Rep 2008; 10:352358.

82 Bentall RP: Madness Explained. Psychosis and Human Nature. London, Penguin, 2005.

83 Bentall RP, Corcoran R, Howard R, Blackwood N, Kinderman P: Persecutory delusions: a review and theoretical integration. Clin Psychol Rev 2001;21:1143-1192.

-84 Zimmermann G, Favrod J, Trieu VH, Pomi$\mathrm{ni} \mathrm{V}$ : The effect of cognitive behavioral treatment on the positive symptoms of schizophrenia spectrum disorders: a meta-analysis. Schizophr Res 2005;77:1-9.

$>85$ Startup M, Jackson MC, Evans KE, Bendix $S$ : North Wales randomized controlled trial of cognitive behaviour therapy for acute schizophrenia spectrum disorders: two-year follow-up and economic evaluation. Psychol Med 2005;35:1307-1316.

- 86 Turkington D, Sensky T, Scott J, Barnes TR, Nur U, Siddle R, Hammond K, Samarasekara N, Kingdon D: A randomized controlled trial of cognitive-behavior therapy for persistent symptoms in schizophrenia: a fiveyear follow-up. Schizophr Res 2008;98:1-7.

87 Klingberg S, Wittorf A, Herrlich J, Wiedemann G, Meisner C, Buchkremer G, Wolwer W: Kognitive Verhaltenstherapie bei Negativsymptomatik schizophrener Störungen. DGPPN Kongress, Berlin, 2009.

- 88 Klingberg S, Wittorf A, Herrlich J, Wiedemann G, Meisner C, Buchkremer G, Frommann N, Wolwer W: Cognitive behavioural treatment of negative symptoms in schizophrenia patients: study design of the TONES study, feasibility and safety of treatment. Eur Arch Psychiatry Clin Neurosci 2009; 259(suppl 2):S149-S154. 